\title{
Self-Reported Sleep Bruxism and Nocturnal Gastroesophageal Reflux Disease in Patients with Obstructive Sleep Apnea: Relationship to Gender and Ethnicity ${ }^{\$}$
}

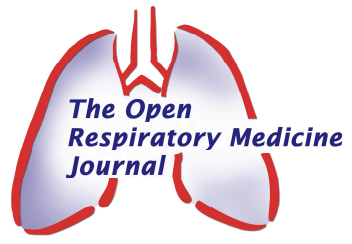

Sean Hesselbacher ${ }^{1,2}$, Shyam Subramanian ${ }^{3}$, Shweta Rao ${ }^{4}$, Lata Casturi ${ }^{4}$ and Salim Surani ${ }^{*}, 5$

${ }^{1}$ Sentara Healthcare, Virginia Beach, VA, USA

${ }^{2}$ Eastern Virginia Medical School, Norfolk, VA, USA

${ }^{3}$ Mercy Health System, Cincinnati, Ohio, USA

${ }^{4}$ Baylor College of Medicine, Houston, Texas, USA

${ }^{5}$ Texas A\&M University, Texas, USA

\begin{abstract}
Study Objectives: Nocturnal bruxism is associated with gastroesophageal reflux disease (GERD), and GERD is strongly associated with obstructive sleep apnea (OSA). Gender and ethnic differences in the prevalence and clinical presentation of these often overlapping sleep disorders have not been well documented. Our aim was to examine the associations between, and the symptoms associated with, nocturnal GERD and sleep bruxism in patients with OSA, and to examine the influence of gender and ethnicity.

Methods: A retrospective chart review was performed of patients diagnosed with OSA at an academic sleep center. The patients completed a sleep questionnaire prior to undergoing polysomnography. Patients with confirmed OSA were evaluated based on gender and ethnicity. Associations were determined between sleep bruxism and nocturnal GERD, and daytime sleepiness, insomnia, restless legs symptoms, and markers of OSA severity in each group.

Results: In these patients with OSA, the prevalence of nocturnal GERD (35\%) and sleep bruxism (26\%) were higher than the general population. Sleep bruxism was more common in Caucasians than in African Americans or Hispanics; there was no gender difference. Nocturnal GERD was similar among all gender and ethnic groups. Bruxism was associated with nocturnal GERD in females, restless legs symptoms in all subjects and in males, sleepiness in African Americans, and insomnia in Hispanics. Nocturnal GERD was associated with sleepiness in males and African Americans, insomnia in females, and restless legs symptoms in females and in Caucasians.

Conclusion: Patients with OSA commonly have comorbid sleep bruxism and nocturnal GERD, which may require separate treatment. Providers should be aware of differences in clinical presentation among different ethnic and gender groups.
\end{abstract}

Keywords: Gastroesophageal reflux, obstructive sleep apnea, sleep bruxism.

\section{CURRENT KNOWLEDGE/STUDY RATIONALE}

Sleep bruxism and nocturnal gastroesophageal reflux disease are common in patients with obstructive sleep apnea, though gender and ethnic differences in the prevalence and clinical presentation have not been well documented. The purpose of this study was to investigate the symptoms associated with nocturnal GERD and bruxism in patients with OSA, and to examine the influence of gender and ethnicity.

\footnotetext{
*Address correspondence to this author at the 7613 Lake Bolsena, Corpus Christi, Texas 78413, USA; Tel: 1-361-885-7722; Fax: 1-361-850-7563; Email: srsurani@hotmail.com
}

${ }^{\S}$ This material was presented in part at the American College of Chest Physicians Annual Meeting, November 2009, San Diego, CA, and at the American Professional Sleep Societies meeting, June 2014, Minneapolis, $\mathrm{MN}$, USA.

\section{STUDY IMPACT}

Significant ethnic and gender differences were identified in the prevalence of sleep bruxism and nocturnal GERD in patients with OSA, and in the frequency of associated sleep disorders such as insomnia, daytime sleepiness, and restless legs symptoms. Such differences should be considered when individualizing diagnostic and treatment plans for these patients.

\section{INTRODUCTION}

Obstructive sleep apnea (OSA) is a common disease with prevalence ranging between $2-7 \%$ in the middle age population with apnea hypopnea index $(\mathrm{AHI})>5$, and is even higher among elderly and in patients with cardiovascular complications and metabolic syndrome [1-4]. 
OSA is characterized by recurrent airway obstruction lasting for longer than 10 seconds during sleep. Whereas, the hypopnea is defined as the drop in peak signal excursion $\geq$ $30 \%$ of the baseline for $\geq 10$ seconds in association with $\geq$ $3 \%$ oxygen desaturation or an arousal [5]. Gastroesophageal reflux disease (GERD) is very prevalent among the general population, with approximately $20 \%$ of the adults experiencing one or more heartburn symptoms per week [6]. More than $70 \%$ of a large survey mentioned nighttime heartburn as a symptom and the majority of them reported sleeping difficulties with heartburn [7].

Bruxism is characterized by stereotypical rhythmic movement of muscles of mastication leading to grinding and clenching of teeth [8]. It is commonly aggravated by stress, sleep disorders, GERD and medications [9, 10]. Sleep bruxism has been associated with arousal response [11] and has been reported to overlap with GERD in many patients [10]. Studies have shown a positive correlation between sleep disordered breathing and tooth grinding [12-14]. There has been mounting evidence of association between GERD and OSA, as well as bruxism and GERD. Gender and ethnic differences in these overlapping conditions have not been looked at. We attempted to examine the associations between, and the symptoms associated with, self-reported GERD and bruxism in patients with OSA, and to examine the influence of gender and ethnicity.

\section{METHODS}

A retrospective review was performed after approval by the IRB of the Baylor College of Medicine. Patients referred for evaluation at the Baylor College of Medicine Sleep Center in Houston, Texas between 2008 and 2009 were reviewed. Inclusion criteria were a diagnosis of obstructive sleep apnea (OSA), defined by an apnea-hypopnea index $(\mathrm{AHI})>5$ events per hour, and age $>18$ years. Records were excluded if the questionnaires were not completed, or if the technical quality or total sleep time during polysomnography (PSG) were inadequate to determine a diagnosis of OSA. Fifty consecutive records in each demographic category (Caucasian males, African American males, Hispanic males, Caucasian females, African American females, and Hispanic females) that met inclusion criteria were selected for review.

\section{Questionnaires and Clinical Assessment}

Each patient completed demographic (including selfreported age, gender, and ethnicity) and sleep-specific (including Epworth Sleepiness Scale [15], and self-reported sleep bruxism, nocturnal GERD, insomnia, and restless legs) questionnaires. The patients then had a consultation with a sleep medicine specialist before undergoing an overnight sleep study for clinical suspicion of OSA or other related health consequences.

\section{Polysomnography}

Sleep studies were performed using attended comprehensive PSG and positive airway pressure, including recording electroencephalogram, electrooculograms, submentalis electromyogram, airflow, respiratory effort, oxygen saturation, anterior tibialis electromyogram, and heart rhythm. Recordings were scored by a technologist manually according to the American Academy of Sleep Medicine Scoring Guidelines [16].

\section{Statistics}

Comparisons between the means of 2 normally distributed groups were performed with the unpaired t-test. Comparisons between 2 non-normally distributed groups were done with the Mann-Whitney U test. Comparisons among more than 2 non-normally distributed groups were performed with the Kruskall-Wallis test. Two groups of dichotomous variables were compared with the Fisher's exact test. A P-value of $<0.05$ was considered statistically significant.

\section{RESULTS}

\section{Demographic and Clinical Characteristics}

Demographic data by gender are represented in Table $\mathbf{1}$. In the overall study group, GERD was reported in $35 \%$ and bruxism in $26 \%$. Both males and females in this cohort had significant morbid obesity with a mean BMI of $41 \pm 9$ in males and $45 \pm 9$ in females. The average AHI for both males $(52.7 \pm 38.2)$ and females $(40.9 \pm 36.7)$ fell in the severe category, covering a wide range of disease severity. No differences between genders were seen in symptomatic complaints, including sleepiness (quantified by the ESS), bruxism, GERD, insomnia, or restless legs symptoms. In Table 2, the demographic data are compared by ethnicity. Of note, Hispanic patients had a higher mean AHI $(55.5 \pm 41.2)$ and lower prevalence of bruxism (19\%) than Caucasians (41.9 \pm 36.6 and $35 \%$, respectively). Other objective and subjective data were similar across groups.

\section{Association of Sleep Bruxism and Nocturnal GERD with Subjective Sleep Complaints}

Self-reported sleep bruxism and nocturnal GERD were associated with other sleep disorders and complaints, as shown in Table 3. Bruxism was associated with nocturnal GERD in all participants $(\mathrm{P}=0.008)$ and females $(\mathrm{P}=0.01)$. Hispanics with bruxism were less likely to report insomnia than those without $(\mathrm{P}=0.047)$. Bruxism and restless legs symptoms were significantly associated in all participants ( $\mathrm{P}$ $=0.01)$, males $(\mathrm{P}=0.009)$, and Caucasian males $(\mathrm{P}=0.02)$. All Caucasians, African American males, Hispanic males, Caucasian females, and Hispanic females did not show any significant associations between bruxism and other sleep disorders/complaints.

GERD was significantly associated with insomnia complaints in all patients $(P=0.007)$, females $(P=0.004)$, and African American females $(P=0.02)$. GERD and restless legs symptoms were significantly associated in females $(P=0.0499)$, Caucasians $(P=0.03)$, and Caucasian females $(\mathrm{P}=0.01)$.

As seen in Fig. (1a), the ESS score was significantly higher in African Americans with bruxism than it was in those without bruxism $(16.0 \pm 5.8$ vs $13.1 \pm 6.2, \mathrm{P}=0.036)$. 
Table 1. Demographics and clinical characteristics by gender.

\begin{tabular}{|l|c|c|c|}
\hline & Males & Females & P-Value \\
\hline \hline Number & 150 & 150 & $<1.7 \pm 9.5$ \\
\hline Age (years), mean \pm s.d. & $46.8 \pm 10.8$ & $1.63 \pm 0.08$ & $<0.0001$ \\
\hline Height (m), mean \pm s.d. & $1.75 \pm 0.09$ & $119.4 \pm 29.6$ & n.s. \\
\hline Weight (kg), mean \pm s.d. & $125.6 \pm 32.3$ & $45.1 \pm 9.8$ & 0.0002 \\
\hline BMI (kg/m), mean \pm s.d. & $41.1 \pm 9.4$ & $13.5 \pm 5.9$ & n.s. \\
\hline ESS, mean \pm s.d. & $13.5 \pm 5.8$ & $40.9 \pm 36.7$ & 0.003 \\
\hline AHI (events/hour), mean \pm s.d. & $52.7 \pm 38.2$ & $72.3 \pm 15.2$ & n.s. \\
\hline SpO nadir (\%), mean \pm s.d. & $71.5 \pm 14.0$ & $34(23 \%)$ & n.s. \\
\hline Bruxism, number (\%) & $43(29 \%)$ & $52(35 \%)$ & n.s. \\
\hline GERD, number (\%) & $53(35 \%)$ & $127(85 \%)$ & n.s. \\
\hline Insomnia, number (\%) & $120(80 \%)$ & $53(35 \%)$ & n.s. \\
\hline RL symptoms, number (\%) & $55(37 \%)$ & \\
\hline
\end{tabular}

s.d. = standard deviation; $\mathrm{m}=$ meters; $\mathrm{kg}=$ kilograms; $\mathrm{n.s.}=$ not significant; BMI = body mass index; ESS =Epworth Sleepiness Scale score; AHI = apnea-hypopnea index $\mathrm{SpO}_{2}=$ oxygen saturation; $\%$ = percentage; $\mathrm{RL}=$ restless legs.

Table 2. Demographics and clinical characteristics by ethnicity.

\begin{tabular}{|c|c|c|c|c|}
\hline & Caucasian & African American & Hispanic & P-Value \\
\hline Number & 100 & 100 & 100 & \\
\hline Height $(m)$, mean \pm s.d. & $1.70 \pm 0.11$ & $1.71 \pm 0.11$ & $1.65 \pm 0.08$ & $<0.0001^{*}$ \\
\hline Weight $(\mathrm{kg})$, mean \pm s.d. & $128.9 \pm 31.9$ & $125.7 \pm 31.6$ & $112.9 \pm 27.6$ & $0.0005^{*}$ \\
\hline ESS, mean \pm s.d. & $13.5 \pm 5.1$ & $13.8 \pm 6.2$ & $13.2 \pm 6.2$ & n.s. \\
\hline AHI (events/hour), mean \pm s.d. & $41.9 \pm 36.6$ & $43.0 \pm 34.2$ & $55.5 \pm 41.2$ & $0.028 * *$ \\
\hline $\mathrm{SpO}_{2}$ nadir $(\%)$, mean \pm s.d. & $73.5 \pm 13.5$ & $72.9 \pm 13.8$ & $68.5 \pm 17.5$ & n.s. \\
\hline Bruxism, number (\%) & $35(35 \%)$ & $23(23 \%)$ & $19(19 \%)$ & $0.026^{* *}$ \\
\hline
\end{tabular}

s.d. = standard deviation; $\mathrm{m}$ = meters; $\mathrm{kg}$ = kilograms; n.s. = not significant; $\mathrm{BMI}=$ body mass index; $\mathrm{ESS}=$ Epworth Sleepiness Scale score; $\mathrm{AHI}=$ apnea-hypopnea index; $\mathrm{SpO} \mathrm{2}_{2}=$ oxygen saturation; $\%=$ percentage; $\mathrm{RL}=$ restless legs.

*Significant differences: Caucasian $v s$ Hispanic, African American vs Hispanic.

**Significant difference: Caucasian vs Hispanic.

This was the only group or subgroup for which bruxism demonstrated a significant association with the ESS. Demonstrated in Fig. (1b), the ESS score was significantly higher in all participants $(14.6 \pm 5.5$ vs $12.9 \pm 5.9, \mathrm{P}=$ $0.020)$, males $(14.9 \pm 5.4$ vs $12.8 \pm 5.9, \mathrm{P}=0.040)$, and African Americans $(16.1 \pm 5.2$ vs $12.2 \pm 6.4, \mathrm{P}=0.002)$ with GERD than those without GERD, with the largest difference being seen in African Americans.

\section{Association of Sleep Bruxism and Nocturnal GERD with Severity of Sleep-Disordered Breathing}

Measures of OSA severity from the overnight PSG were compared with the presence of sleep bruxism and nocturnal
GERD. The mean AHI (Fig. 2a) and $\mathrm{SpO}_{2}$ nadir (Fig. 2b) were similar between those with and without sleep bruxism, in all groups and subgroups. Likewise, those that reported nocturnal GERD did not differ in AHI (Fig. 2c) or $\mathrm{SpO}_{2}$ nadir (Fig. 2d) from those that did not report GERD. However, African American males with GERD had a higher AHI $(65.8 \pm 38.8$ vs $45.1 \pm 35.0 ; \mathrm{P}=0.04)$ and lower $\mathrm{SpO} 2$ nadir $(68.2 \% \pm 15.2 \%$ vs $74.4 \% \pm 13.2 \% ; \mathrm{P}=0.04)$ than those without, data not shown. No other subgroups demonstrated any significant associations.

\section{DISCUSSION}

These data show for the first time some of the differences in symptoms reported by various gender and ethnic groups 
Table 3. Correlation of sleep bruxism and nocturnal GERD with other sleep-related factors and complaints vary among ethnic and gender groups and subgroups.

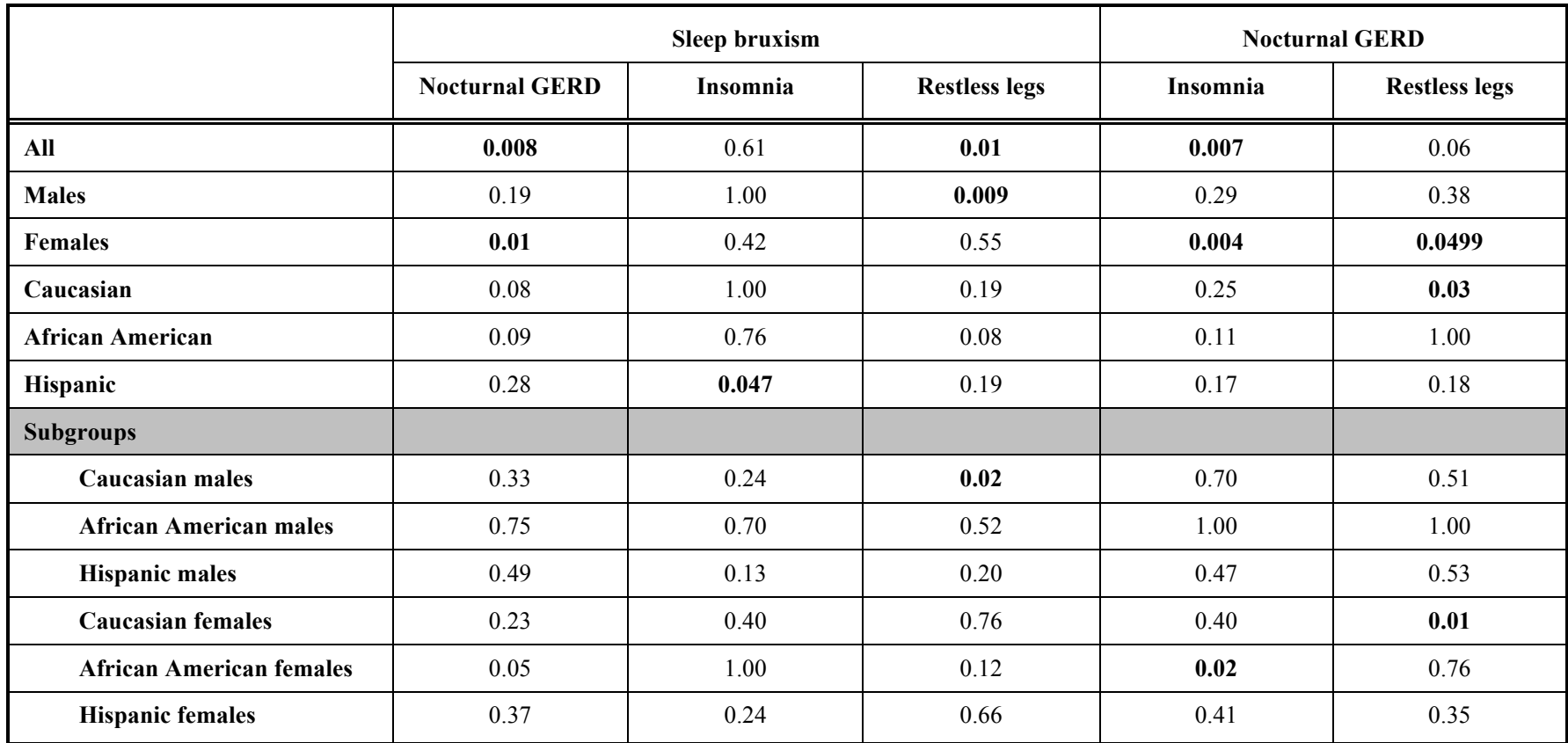

Data reported as P-values by Fisher's exact test (bold indicates $\mathrm{P}<0.05)$.
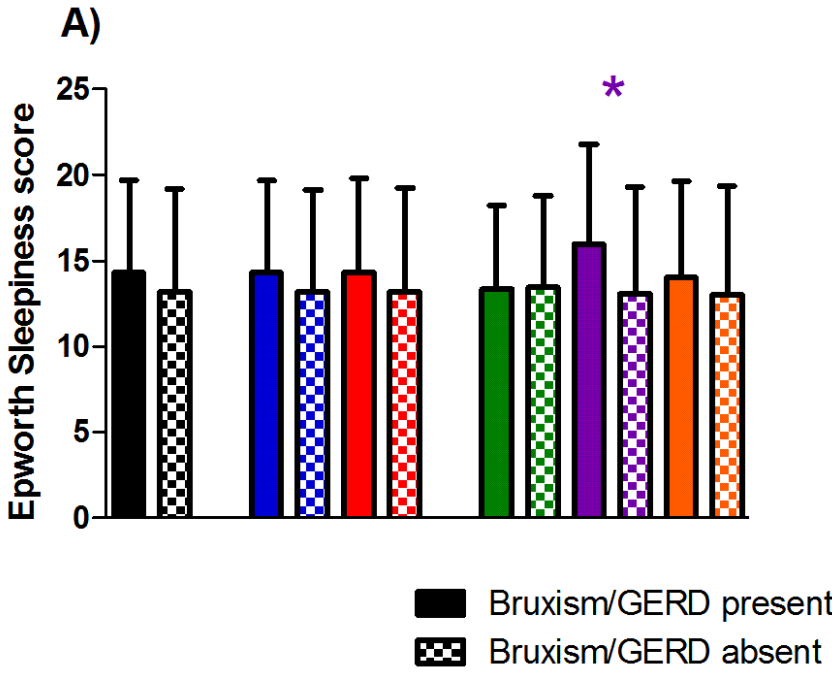

B)

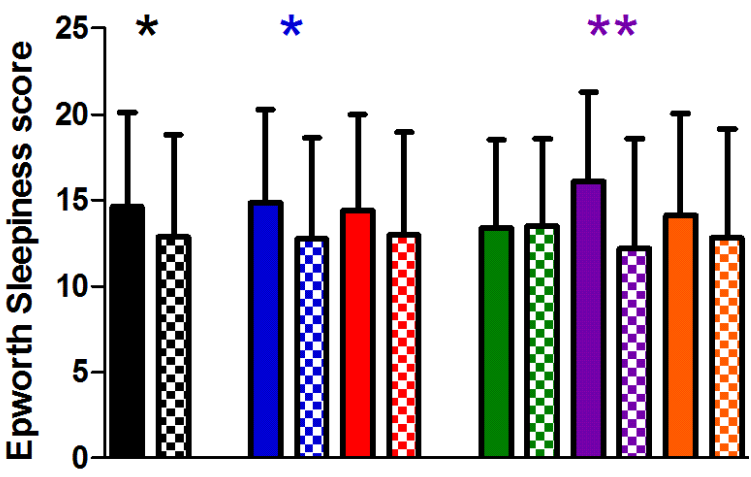

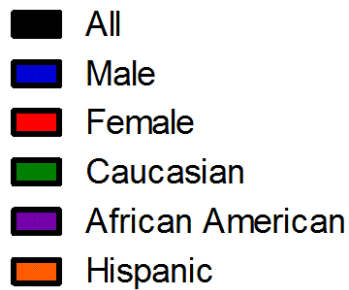

Fig. (1). The Epworth Sleepiness score is higher in some patients with sleep-related bruxism and nocturnal GERD. The mean and standard deviation are shown for patients with (solid bar) and without (checkered bar) bruxism (A) and GERD (B). Subgroups by gender and ethnicity are identified by color. ${ }^{*} \mathrm{P}<0.05,{ }^{*} * \mathrm{P}<0.01$.

with sleep apnea in association with sleep-related bruxism and nocturnal GERD. While African Americans reported significantly more daytime sleepiness with bruxism, Hispanics less often reported insomnia. In agreement with prior studies $[10,17]$, patients with bruxism often reported nocturnal GERD. Caucasians with bruxism most often reported GERD, though ethnicity alone did not explain the association with bruxism. Females had the highest association between these two sleep-related symptoms; there was a trend toward an association in African American females specifically. Sleep bruxism was not associated with indicators of OSA severity, similar to previously reported data $[14,18]$.

Like bruxism, the presence of GERD was not associated with markers of OSA severity, except in African American 
A)

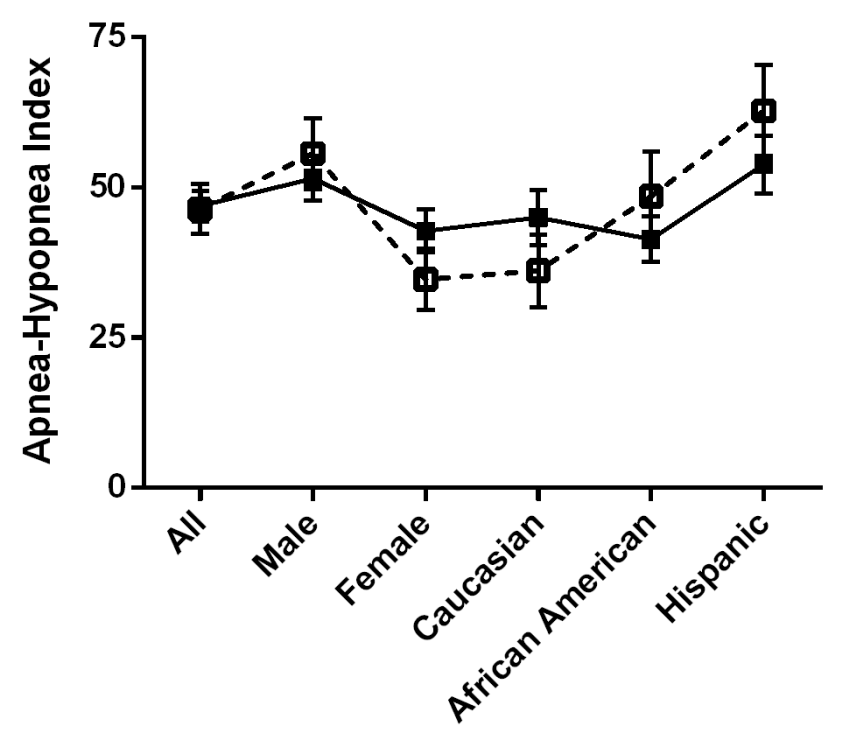

C)

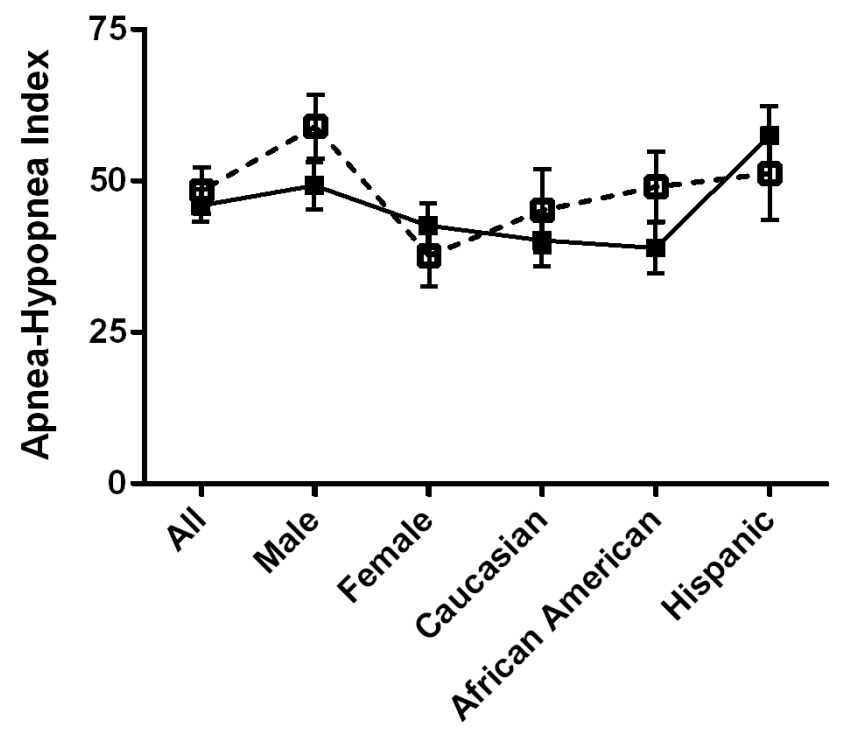

B)

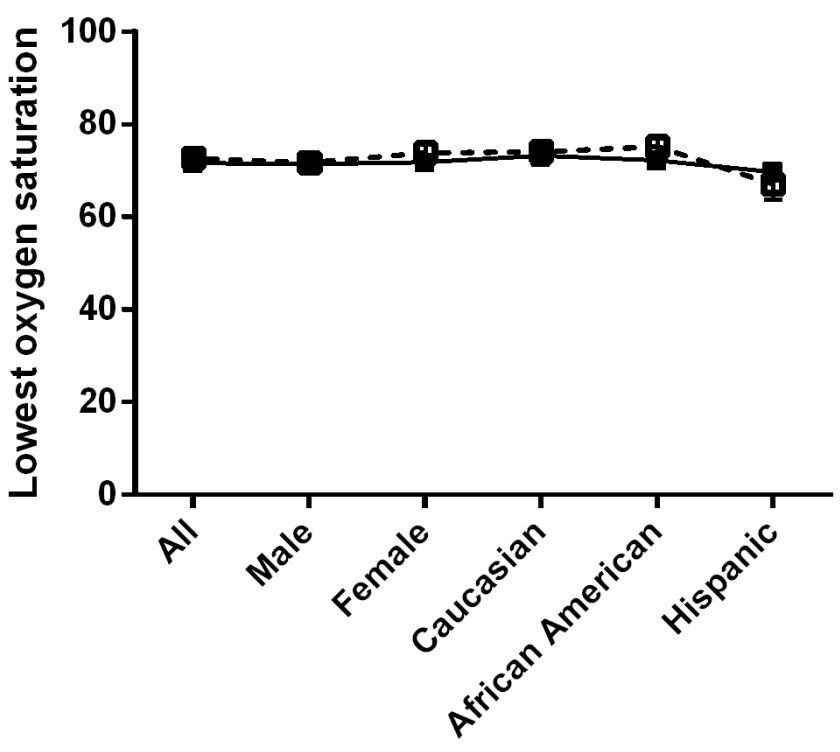

D)

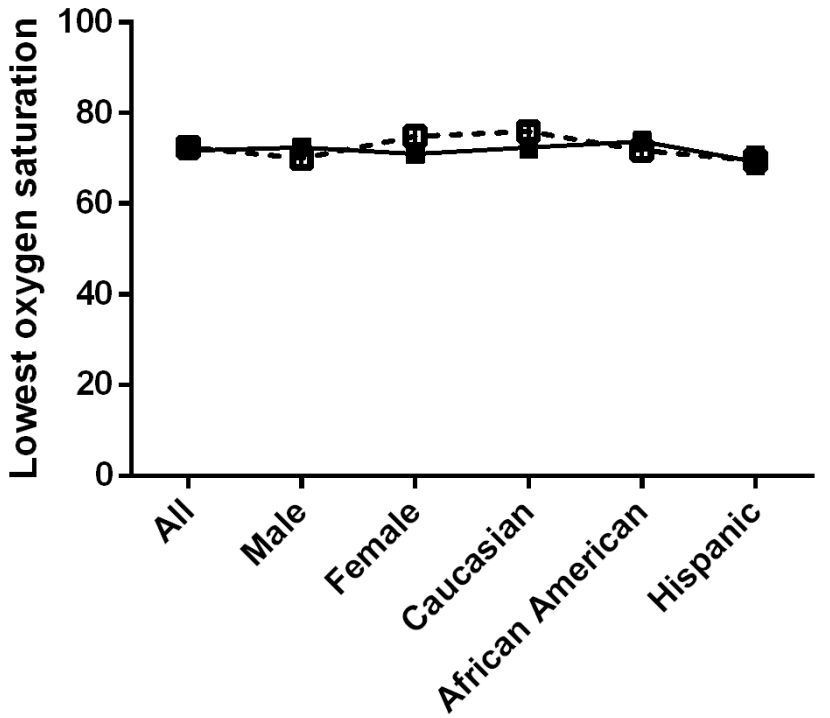

Fig. (2). The severity of obstructive sleep apnea (OSA) is not associated with the presence of with sleep-related bruxism or nocturnal GERD in patients with OSA. The mean and standard error of the mean of the apnea-hypopnea index $(\mathbf{A}, \mathbf{C})$ and lowest oxygen saturation $(\mathbf{B}, \mathbf{D})$ are shown for patients with (solid box, solid line) and without (open box, dashed line) bruxism (A, B) and GERD (C, D).

males. Insomnia was a common complaint in patients with nocturnal GERD; this was especially the case in females and African American females. Sleepiness was also more common in nocturnal GERD, primarily in males and African Americans. The prevalence of restless legs symptoms was similar in all ethnic groups studied, though the symptoms were correlated with sleep bruxism in Caucasian males and with nocturnal GERD in Caucasian females. Sleep bruxism and nocturnal GERD were both more prevalent in our patients with OSA than in the general population $[19,20]$.

Both sleep-related bruxism and nocturnal GERD have the potential to disrupt sleep and lead to either daytime sleepiness or insomnia. The factors behind an individual or group response to a given sleep disorder are innumerable and will take much more study to delineate. Reported symptoms of sleep-disordered breathing are known to vary across ethnicities and genders [21-24]. It is only when the ethnic and gender subgroups are looked at more closely that we start to see stronger associations, thus highlighting the potentially important differences in clinical presentation among the groups.

As the practice of sleep medicine moves out of the sleep laboratory, and sometimes out of the sleep center entirely, history-taking and personalization of the treatment regimen become more important. Medications and simple diagnostic criteria allow evaluation and treatment of RLS, OSA, and insomnia in the primary care or subspecialist settings, without the use of polysomnography. Without the knowledge of associated or contributing factors, and without the allotted time to perform a thorough sleep review of symptoms, more subtle findings of nocturnal GERD and sleep-related bruxism may go unnoticed and untreated. Cultural or 
language barriers that inhibit medical history taking could also influence the likelihood of detecting these problems. Insomnia, GERD, and bruxism all have known short- and long-term health consequences, thus magnifying the importance of appropriate diagnosis and treatment.

Nocturnal GERD is often associated with sleep-related bruxism. Case reports have shown that bruxism, and the associated sleep disturbances, improve with treatment of OSA $[13,25]$. Previous data has shown that continuous positive airway pressure reduced objective measures of GERD in subjects with or without OSA [26]. Our study did not investigate the effect of OSA treatment on bruxism or GERD, though this may also vary by ethnicity or gender. OSA is thought to contribute to bruxism through high levels of sympathetic activity or by increasing the frequency of arousals. GERD has long been associated with OSA. It has been postulated that the negative intra-thoracic pressure generated during apneic episodes can predispose someone with OSA to increased reflux [27]. Even mild OSA may result in markedly disrupted sleep or generate negative intrathoracic pressure, so it might be expected that the severity, as measured by $\mathrm{AHI}$ and $\mathrm{SpO}_{2}$ nadir, did not correlate with the bruxism or GERD complaints. Obesity is often a contributor to both OSA and GERD. The mean BMI of the patients studied was in the range of morbidly obese in all ethnicities and genders; additionally, the mean AHI fell into the category of severe OSA. These factors likely reflect local referral patterns and may play a role in the proportion of patients that have related comorbidities, such as nocturnal GERD. No association was seen between BMI and GERD complaint in our cohort $(\mathrm{P}=0.36)$. Among the 16 non-obese patients included our analyses, $6(37.5 \%)$ reported nocturnal GERD and 9 (56.3\%) reported sleep bruxism; the prevalence of GERD in this small subgroup was similar to the overall study population, though bruxism was much more common. The association of obesity with sleep-related complaints in patients with OSA is another area of future research using an appropriate study population.

Obstructive sleep apnea presents in a variety of ways, with known ethnic and gender variations. Because our cohort was comprised entirely of patients with OSA, it is unknown if our results are applicable to patients without OSA. Since many patients with non-apnea sleep problems are treated in the primary care setting [28-31], application of our findings to the at-large community will be an important next step; future studies should be directed toward this goal. Furthermore, our data and the work of others, have shown that many sleep disorders occur more commonly in patients with OSA; therefore, there should be a low threshold to evaluate for OSA in patients presenting with these symptoms.

Other limitations to the present study include the methods by which the sleep disorders were detected. In order to encourage participation by patients undergoing PSG, the questionnaires were truncated. Because formal diagnostic criteria were not employed, identification of restless legs symptoms in this case is not equivalent to the presence of restless legs syndrome. Likewise, the severity and frequency of the symptoms (GERD, bruxism, insomnia, and restless legs) were not taken into consideration; the participants were asked to mark a box if the symptoms were present. While the threshold to respond is different for each participant, it is presumed that an affirmative response reflects a minimum symptom severity and frequency to have an impact on the patient in a meaningful way. Detection of bruxism by electroencephalographic artifact, GERD by $\mathrm{pH}$ probe, or quantification of periodic limb movements during sleep as a surrogate of RLS would provide objective measures of these comorbidities, but would not assess the clinical impact. Also, bruxism and RLS are diagnosed by clinical criteria; PSG features are neither sufficient nor necessary. A small study of 18 bruxers reported frequent tooth-grinding during sleep, with tooth wear noted in large majority of them; asymptomatic controls did not exhibit these findings. Additionally, those reporting bruxism had significantly more frequent bruxism events recorded during PSG [32]. In contrast, GERD symptoms do not correlate as well with objective data. GERD symptoms, including nocturnal heartburn, were compared with 24 -hour $\mathrm{pH}$ probe in 336 patients. Nocturnal heartburn was independently associated with abnormal findings on $\mathrm{pH}$ probe; however, $51 \%$ of patients with severe GERD symptoms did not have findings consistent with pathologic GERD [33]. Our study was performed at a single sleep center, which maintains consistency in scoring and implementation of the tests, but the ethnic groups in the study groups (Caucasians, Hispanics, and African Americans) were limited to the local population. Given the absence of participants from other ethnicities, the conclusions are not necessarily applicable to these other groups.

The number of associated sleep disorders evaluated was purposefully limited in our study; the intention was to focus on clinically meaningful associations and symptoms. However, any number of sleep-related symptoms can potentially be worsened by sleep-related bruxism or nocturnal GERD. Similarly, the presence of other conditions, including medical comorbidities or substance abuse, can be expected to exacerbate these sleep problems. Of note, a recent systematic review did not find a relationship between psychosocial disorders and sleep bruxism [9]. Future research may be geared towards identifying other important associations with these sleep disorders, further advancing our understanding of common mechanisms and defining at-risk populations.

\section{CONCLUSION}

Both sleep bruxism and nocturnal GERD are common in patients with OSA. These symptoms may overlap in the same patient or present in conjunction with other sleep disorders, all of which may require treatment. Differences in symptom presentation between ethnic and gender groups can make identification of these sleep disorders more difficult; however, understanding these differences may help providers more effectively treat a variety of patient populations.

\section{CONFLICT OF INTEREST}

The authors confirm that this article content has no conflict of interest.

\section{ACKNOWLEDGEMENTS}

Declared none. 


\section{REFERENCES}

[1] Ding N, Ni BQ, Zhang XL, et al. Prevalence and risk factors of sleep disordered breathing in patients with rheumatic valvular heart disease. J Clin Sleep Med 2013; 9: 781-7.

[2] Monahan K, Redline S. Role of obstructive sleep apnea in cardiovascular disease. Curr Opin Cardiol 2011; 26: 541-7.

[3] Punjabi NM, Caffo BS, Goodwin JL, et al. Sleep-disordered breathing and mortality: a prospective cohort study. PLoS Med 2009; 6: e1000132.

[4] Young T, Peppard PE, Gottlieb DJ. Epidemiology of obstructive sleep apnea: a population health perspective. Am J Respir Crit Care Med 2002; 165: 1217-39.

[5] Berry RB, Budhiraja R, Gottlieb DJ, et al. Rules for scoring respiratory events in sleep: update of the 2007 AASM manual for the scoring of sleep and associated events. deliberations of the sleep apnea definitions task force of the American academy of sleep medicine. J Clin Sleep Med 2012; 8: 597-619.

[6] Locke GR, 3rd. Current medical management of gastroesophageal reflux disease. Thorac Surg Clin 2005; 15: 369-75.

[7] Shaker R, Castell DO, Schoenfeld PS, et al. Nighttime heartburn is an under-appreciated clinical problem that impacts sleep and daytime function: the results of a Gallup survey conducted on behalf of the American Gastroenterological Association. Am J Gastroenterol 2003; 98: 1487-93.

[8] AASM. International classification of sleep disorders, revised. Diagnostic and coding manual: American Academy of Sleep Medicine, $2^{\text {nd }}$ ed. 2005: 4.

[9] Manfredini D, Lobbezoo F. Role of psychosocial factors in the etiology of bruxism. J Orofac Pain 2009; 23: 153-66.

[10] 10 Mengatto CM, Dalberto Cda S, Scheeren B, Barres SG. Association between sleep bruxism and gastroesophageal reflux disease. J Prosthet Dent 2013; 110: 349-55.

[11] Sato T, Harada Y. Tooth-grinding during sleep as an arousal reaction. Experientia 1971; 27: 785-6.

[12] Phillips BA, Okeson J, Paesani D, et al. Effect of sleep position on sleep apnea and parafunctional activity. Chest 1986; 90: 424-29.

[13] Oksenberg A, Arons E. Sleep bruxism related to obstructive sleep apnea: the effect of continuous positive airway pressure. Sleep Med 2002; 3: 513-5.

[14] Sjoholm TT, Lowe AA, Miyamoto K, et al. Sleep bruxism in patients with sleep-disordered breathing. Arch Oral Biol 2000; 45: 889-96.

[15] Johns MW. A new method for measuring daytime sleepiness: the Epworth sleepiness scale. Sleep 1991; 14: 540-5.

[16] Grigg-Damberger M, Gozal D, Marcus CL, et al. The visual scoring of sleep and arousal in infants and children. J Clin Sleep Med 2007; 3: 201-40.
[17] Miyawaki S, Tanimoto Y, Araki Y, et al. Association between nocturnal bruxism and gastroesophageal reflux. Sleep 2003; 26: 888-92.

[18] Ohayon MM, Li KK, Guilleminault C. Risk factors for sleep bruxism in the general population. Chest 2001; 119: 53-61.

[19] Zhao WE. Statistical Brief \#44. Healthcare Cost and Utilization Project (HCUP). December 2007. : Agency for Healthcare Research and Quality, Rockville MD 2013.

[20] Manfredini D, Winocur E, Guarda-Nardini L, et al. Epidemiology of bruxism in adults: a systematic review of the literature. J Orofac Pain 2013; 27: 99-110.

[21] O'Connor GT, Lind BK, Lee ET, et al. Variation in symptoms of sleep-disordered breathing with race and ethnicity: the Sleep Heart Health Study. Sleep 2003; 26: 74-9.

[22] Valipour A, Lothaller H, Rauscher H, et al. Gender-related differences in symptoms of patients with suspected breathing disorders in sleep: a clinical population study using the sleep disorders questionnaire. Sleep 2007; 30: 312-9.

[23] Hesselbacher S, Subramanian S, Allen J, et al. Body mass index, gender, and ethnic variations alter the clinical implications of the epworth sleepiness scale in patients with suspected obstructive sleep apnea. Open Respir Med J 2012; 6: 20-7.

[24] Subramanian S, Guntupalli B, Murugan T, et al. Gender and ethnic differences in prevalence of self-reported insomnia among patients with obstructive sleep apnea. Sleep Breath 2011; 15: 711-5.

[25] Hamada S, Chin K, Hitomi T, et al. Impact of nasal continuous positive airway pressure for congenital adrenal hyperplasia with obstructive sleep apnea and bruxism. Sleep Breath 2012; 16: 11-5.

[26] Ing AJ, Ngu MC, Breslin ABX. Obstructive sleep apnea and gastroesophageal reflux. Am J Med 2000; 108: 120-5.

[27] Gaude GS. Pulmonary manifestations of gastroesophageal reflux disease. Ann Thorac Med 2009; 4: 115-23.

[28] Silber MH, Becker PM, Earley C, et al. Willis-Ekbom Disease Foundation revised consensus statement on the management of restless legs syndrome. Mayo Clin Proc 2013; 88: 977-86.

[29] Nichols DA, Allen RP, Grauke JH, et al. Restless legs syndrome symptoms in primary care: a prevalence study. Arch Intern Med 2003; 163: 2323-9.

[30] Krakow B, Ulibarri VA, Romero EA, et al. A two-year prospective study on the frequency and co-occurrence of insomnia and sleepdisordered breathing symptoms in a primary care population. Sleep Med 2013; 14: 814-23.

[31] Falloon K, Arroll B, Elley CR, et al. The assessment and management of insomnia in primary care. BMJ 2011;342: d2899.

[32] Lavigne GJ, Rompre PH, Montplaisir JY. Sleep bruxism: validation of clinical research diagnostic criteria in a controlled polysomnographic study. J Dent Res 1996; 75: 546-52.

[33] Chan K, Liu G, Miller L, et al. Lack of correlation between a selfadministered subjective GERD questionnaire and pathologic GERD diagnosed by 24-h esophageal $\mathrm{pH}$ monitoring. J Gastrointest Surg 2010; 14: 427-36.

(C) Hesselbacher et al.; Licensee Bentham Open.

This is an open access article licensed under the terms of the Creative Commons Attribution Non-Commercial License (http://creativecommons.org/licenses/by-nc/3.0/) which permits unrestricted, non-commercial use, distribution and reproduction in any medium, provided the work is properly cited. 